\title{
HOMOCLINIC ORBITS FOR A CLASS OF DISCRETE PERIODIC HAMILTONIAN SYSTEMS
}

\author{
QINQIN ZHANG
}

(Communicated by Yingfei Yi)

\begin{abstract}
In this paper we establish new criteria for the existence of nontrivial homoclinic orbits to a class of discrete Hamiltonian systems. Our results do not need to suppose that the system satisfies the well-known global Ambrosetti-Rabinowitz superquadratic assumption.
\end{abstract}

\section{INTRODUCTION}

Consider the second-order self-adjoint discrete Hamiltonian system

$$
\triangle[p(n) \triangle u(n-1)]-L(n) u(n)+\nabla W(n, u(n))=0,
$$

where $n \in \mathbb{Z}, u \in \mathbb{R}^{\mathcal{N}}, \triangle u(n)=u(n+1)-u(n)$ is the forward difference, $p, L$ : $\mathbb{Z} \rightarrow \mathbb{R}^{\mathcal{N} \times \mathcal{N}}$ and $W: \mathbb{Z} \times \mathbb{R}^{\mathcal{N}} \rightarrow \mathbb{R}$ satisfy

(P) $p(n)$ is an $\mathcal{N} \times \mathcal{N}$ real symmetric positive definite matrix for all $n \in \mathbb{Z}$, and $p(n+N)=p(n), \forall n \in \mathbb{Z}$;

(L) $L(n)$ is an $\mathcal{N} \times \mathcal{N}$ real symmetric positive definite matrix for all $n \in \mathbb{Z}$, $L(n+N)=L(n), \forall n \in \mathbb{Z}$, and there exists a constant $\beta>0$ such that

$$
(L(n) x, x) \geq \beta|x|^{2}, \quad \forall(n, x) \in \mathbb{Z} \times \mathbb{R}^{\mathcal{N}} ;
$$

(W1) $W(n, x)$ is continuously differentiable in $x$ for every $n \in \mathbb{Z}, W(n, 0)=0$, $W(n, x) \geq 0$ and $W(n+N, x)=W(n, x)$ for all $(n, x) \in \mathbb{Z} \times \mathbb{R}^{\mathcal{N}}$, where $N$ is a given positive integer;

(W2) $\lim _{|x| \rightarrow 0} \frac{\nabla W(n, x)}{|x|}=0$ uniformly for $n \in \mathbb{Z}$.

In general, system (1.1) may be regarded as a discrete analogue of the following second-order Hamiltonian system

$$
\left[p(t) u^{\prime}(t)\right]^{\prime}-L(t) u(t)+\nabla W(t, u(t))=0
$$

which has been extensively studied in the literature on the existence and multiplicity of its homoclinic orbits (see, for example [6], 10, 11, 16] and references therein). Moreover, system (1.1) also comes from actual applications; see the monographs [1], [2].

Received by the editors November 18, 2012 and, in revised form, December 4, 2012.

2010 Mathematics Subject Classification. Primary 58E05; Secondary 70H05.

This project was supported by the Doctoral Program Foundation of the Ministry of Education of China (20104410110001). 
As usual, we say that a solution $u(n)$ of system (1.1) is homoclinic (to 0) if $u(n) \rightarrow 0$ as $n \rightarrow \pm \infty$. In addition, if $u(n) \not \equiv 0$, then $u(n)$ is called a nontrivial homoclinic solution.

It is well known that homoclinic orbits play a very important role in the study of chaos in dynamical systems. It has been proved that the system must be chaotic provided it has the transversely intersected homoclinic orbits. Therefore, it possesses important theoretical significance and practical value to investigate the existence of homoclinic orbits of system (1.1) emanating from zero.

In recent years, the existence of homoclinic solutions of system (1.1) or their special forms have been investigated by many authors; see, for example [13, 15] where $W(n, x)$ is subquadratic as $|x| \rightarrow \infty$ and [4, [5], 7], 8], 9], 11, 14], 17], 18] where $W(n, x)$ is superquadratic. Moreover, in the superquadratic case, almost all the existing results always need the next well-known global Ambrosetti-Rabinowitz superquadratic condition:

(AR) there exists $\mu>2$ such that

$$
0<\mu W(n, x) \leq(\nabla W(n, x), x), \quad \forall(n, x) \in \mathbb{Z} \times \mathbb{R}^{\mathcal{N}} \backslash\{0\},
$$

where and in the sequel, $(\cdot, \cdot)$ denotes the standard inner product in $\mathbb{R}^{\mathcal{N}}$ and $|\cdot|$ is the induced norm.

In this paper, we introduce the following weaker assumptions than (AR):

(W3) $\lim _{|x| \rightarrow \infty} \frac{|W(n, x)|}{|x|^{2}}=\infty$ for all $n \in \mathbb{Z}$;

(W4) $\widetilde{W}(n, x):=\frac{1}{2}(\nabla W(n, x), x)-W(n, x)>0, \forall(n, x) \in \mathbb{Z} \times\left(\mathbb{R}^{\mathcal{N}} \backslash\{0\}\right)$, and there exist $c_{1}>0$ and $R_{0}>0$ such that

$$
W(n, x) \leq c_{1}|x|^{2} \widetilde{W}(n, x), \quad \forall(n, x) \in \mathbb{Z} \times \mathbb{R}^{\mathcal{N}}, \quad|x| \geq R_{0} .
$$

Now, we are ready to state the main result of this paper.

Theorem 1.1. Assume that $p, L$ and $W$ satisfy (P), (L), (W1), (W2), (W3) and (W4). Then system (1.1) possesses a nontrivial homoclinic solution.

Remark 1.2. If $W(n, x)$ satisfies (AR), then there exists a constant $C_{0}>0$ such that

$$
W(n, x) \geq C_{0}|x|^{\mu}, \quad \forall(n, x) \in \mathbb{Z} \times \mathbb{R}^{\mathcal{N}}, \quad|x| \geq 1 ;
$$

moreover $\widetilde{W}(n, x)>0$ for all $(n, x) \in \mathbb{Z} \times\left(\mathbb{R}^{\mathcal{N}} \backslash\{0\}\right)$, and

$$
W(n, x) \leq \frac{2}{\mu-2}|x|^{2} \widetilde{W}(n, x), \quad \forall(n, x) \in \mathbb{Z} \times \mathbb{R}^{\mathcal{N}}, \quad|x| \geq 1 .
$$

These show that (W3) and (W4) are satisfied.

Remark 1.3. There are many functions $W(n, x)$ that satisfy (W3) and (W4) but not (AR). For example:

$$
\begin{gathered}
W(n, x)=a(n)|x|^{2} \ln (1+|x|), \\
W(n, x)=b(n)\left(|x|^{4}+2|x|^{3} \sin ^{2}|x|\right),
\end{gathered}
$$

where $a(n)$ and $b(n)$ are $N$-periodic positive functions. 


\section{Preliminaries}

Throughout this section, we always assume that $p$ and $L$ satisfy (P) and (L). Let

$$
\begin{gathered}
S=\left\{\{u(n)\}_{n \in \mathbb{Z}}: u(n) \in \mathbb{R}^{\mathcal{N}}, n \in \mathbb{Z}\right\}, \\
E=\left\{u \in S: \sum_{n \in \mathbb{Z}}[(p(n+1) \triangle u(n), \triangle u(n))+(L(n) u(n), u(n))]<+\infty\right\},
\end{gathered}
$$

and for $u, v \in E$, let

$$
\langle u, v\rangle=\sum_{n \in \mathbb{Z}}[(p(n+1) \triangle u(n), \triangle v(n))+(L(n) u(n), v(n))] .
$$

Then $E$ is a Hilbert space with the above inner product, and the corresponding norm is

$$
\|u\|=\left\{\sum_{n \in \mathbb{Z}}[(p(n+1) \triangle u(n), \triangle u(n))+(L(n) u(n), u(n))]\right\}^{1 / 2}, \quad u \in E .
$$

As usual, for $1 \leq q<+\infty$, set

$$
l^{q}\left(\mathbb{Z}, \mathbb{R}^{\mathcal{N}}\right)=\left\{u \in S: \sum_{n \in \mathbb{Z}}|u(n)|^{q}<+\infty\right\}
$$

and

$$
l^{\infty}\left(\mathbb{Z}, \mathbb{R}^{\mathcal{N}}\right)=\left\{u \in S: \sup _{n \in \mathbb{Z}}|u(n)|<+\infty\right\},
$$

and their norms are defined by

$$
\|u\|_{q}=\left(\sum_{n \in \mathbb{Z}}|u(n)|^{q}\right)^{1 / q}, \quad \forall u \in l^{q}\left(\mathbb{Z}, \mathbb{R}^{\mathcal{N}}\right) ; \quad\|u\|_{\infty}=\sup _{n \in \mathbb{Z}}|u(n)|, \quad \forall u \in l^{\infty}\left(\mathbb{Z}, \mathbb{R}^{\mathcal{N}}\right),
$$

respectively. Evidently, $E$ is continuously embedded into $l^{q}\left(\mathbb{Z}, \mathbb{R}^{\mathcal{N}}\right)$ for $2 \leq q \leq \infty$; i.e., there exists $\gamma_{q}>0$ such that

$$
\|u\|_{q} \leq \gamma_{q}\|u\|, \quad \forall u \in E .
$$

Lemma 2.1 ([9]). For $u \in E$, one has

$$
\|u\|_{\infty} \leq \frac{1}{\sqrt[4]{(\beta+4 \alpha) \beta}}\|u\|
$$

where $\alpha=\inf \left\{(p(n) x, x): n \in \mathbb{Z}, x \in \mathbb{R}^{\mathcal{N}},|x|=1\right\}$.

Lemma $2.2([3])$. Let $E$ be a real Banach space with its dual space $E^{*}$ and suppose that $I \in C^{1}(E, \mathbb{R})$ satisfies

$$
\max \{I(0), I(e)\} \leq \eta_{0}<\eta \leq \inf _{\|u\|=\rho} I(u),
$$

for some $\eta_{0}<\eta, \rho>0$ and $e \in E$ with $\|e\|>\rho$. Let $c \geq \eta$ be characterized by

$$
c=\inf _{\gamma \in \Gamma} \max _{0 \leq t \leq 1} I(\gamma(t)),
$$

where $\Gamma=\{\gamma \in C([0,1], E): \gamma(0)=0, \gamma(1)=e\}$ is the set of continuous paths joining 0 to $e$; then there exists $\left\{u_{k}\right\}_{k \in \mathbb{N}} \subset E$ such that

$$
I\left(u_{k}\right) \rightarrow c \text { and }\left(1+\left\|u_{k}\right\|\right)\left\|I^{\prime}\left(u_{k}\right)\right\|_{E^{*}} \rightarrow 0 \text { as } k \rightarrow \infty .
$$


Now we define a functional $\Phi$ on $E$ by

$$
\Phi(u)=\frac{1}{2} \sum_{n \in \mathbb{Z}}[(p(n+1) \triangle u(n), \triangle u(n))+(L(n) u(n), u(n))]-\sum_{n \in \mathbb{Z}} W(n, u(n)) .
$$

For any $u \in E$, there exists an $n_{1} \in \mathbb{N}$ such that $|u(n)| \leq 1$ for $|n| \geq n_{1}$. Hence, under assumptions $(\mathrm{P}),(\mathrm{L}),(\mathrm{W} 1)$ and $(\mathrm{W} 2)$, the functional $\Phi$ is of class $C^{1}(E, \mathbb{R})$. Moreover,

$$
\begin{gathered}
\Phi(u)=\frac{1}{2}\|u\|^{2}-\sum_{n \in \mathbb{Z}} W(n, u(n)), \quad \forall u \in E, \\
\left\langle\Phi^{\prime}(u), v\right\rangle=\langle u, v\rangle-\sum_{n \in \mathbb{Z}}(\nabla W(n, u(n)), v(n)), \quad \forall u, v \in E .
\end{gathered}
$$

Furthermore, the critical points of $\Phi$ in $E$ are solutions of system (1.1) with $u( \pm \infty)=0$; see [9], [13].

Lemma 2.3. Under assumptions (P), (L), (W1), (W2) and (W3), there exist a constant $c>0$ and a sequence $\left\{u_{k}\right\} \subset E$ satisfying

$$
\Phi\left(u_{k}\right) \rightarrow c, \quad\left\|\Phi^{\prime}\left(u_{k}\right)\right\|\left(1+\left\|u_{k}\right\|\right) \rightarrow 0 .
$$

Proof. From (W2), there exists $\delta_{0}>0$ such that

$$
|\nabla W(t, x)| \leq \frac{1}{2 \gamma_{2}^{2}}|x|, \quad \forall(n, x) \in \mathbb{Z} \times \mathbb{R}^{\mathcal{N}}, \quad|x| \leq \delta_{0}
$$

Since $W(n, 0)=0$, it follows that

$$
W(n, x) \leq \frac{1}{4 \gamma_{2}^{2}}|x|^{2}, \quad \forall(n, x) \in \mathbb{Z} \times \mathbb{R}^{\mathcal{N}}, \quad|x| \leq \delta_{0} .
$$

If $\|u\|=\sqrt[4]{(\beta+4 \alpha) \beta} \delta_{0}:=\rho$, then by Lemma $2.1,|u(n)| \leq \delta_{0}$ for all $n \in \mathbb{Z}$. Hence, from (2.4) and (2.7), one has

$$
\begin{aligned}
\Phi(u) & =\frac{1}{2}\|u\|^{2}-\sum_{n \in \mathbb{Z}} W(n, u(n)) \\
& \geq \frac{1}{2}\|u\|^{2}-\frac{1}{4 \gamma_{2}^{2}} \sum_{n \in \mathbb{Z}}|u(n)|^{2} \\
& =\frac{1}{2}\|u\|^{2}-\frac{1}{4 \gamma_{2}^{2}}\|u\|_{2}^{2} \\
& \geq \frac{1}{4}\|u\|^{2}=\frac{1}{4} \rho^{2}, \quad \forall u \in E, \quad\|u\|=\rho .
\end{aligned}
$$

Choose $u_{0} \in E$ as follows:

$$
u_{0}(0)=(1,0, \cdots, 0)^{\top} \in \mathbb{R}^{\mathcal{N}}, \quad u_{0}(n)=0, \quad \forall n \neq 0 .
$$


Then it follows from (W1), (W3) and (2.4) that

$$
\begin{aligned}
\Phi\left(s u_{0}\right) & =\frac{s^{2}}{2}\left\|u_{0}\right\|^{2}-\sum_{n \in \mathbb{Z}} W\left(n, s u_{0}(n)\right) \\
& =\frac{s^{2}}{2}\left\|u_{0}\right\|^{2}-W\left(0, s u_{0}(0)\right) \\
& =s^{2}\left[\frac{1}{2}\left\|u_{0}\right\|^{2}-\frac{W\left(0, s u_{0}(0)\right)}{\left|s u_{0}(0)\right|^{2}}\right] \\
& \leq 0, \quad \text { for large } s>0 .
\end{aligned}
$$

Choose $s_{1}>1$ such that $s_{1}\left\|u_{0}\right\|>\rho$ and $\Phi\left(s_{1} u_{0}\right) \leq 0$. Let $e=s_{1} u_{0}$; then $e \in E$, $\|e\|>\rho$ and $\Phi(e) \leq 0$. By Lemma 2.2, there exist a constant $c \geq \rho^{2} / 4$ and a sequence $\left\{u_{k}\right\} \subset E$ such that (2.6) holds.

Lemma 2.4. Under assumptions (P), (L), (W1), (W2), (W3) and (W4), any sequence $\left\{u_{k}\right\} \subset E$ satisfying

$$
\Phi\left(u_{k}\right) \rightarrow c>0, \quad\left\langle\Phi^{\prime}\left(u_{k}\right), u_{k}\right\rangle \rightarrow 0
$$

is bounded in $E$.

Proof. To prove the boundedness of $\left\{u_{k}\right\}$, arguing by contradiction, we suppose that $\left\|u_{k}\right\| \rightarrow \infty$. Let $v_{k}=u_{k} /\left\|u_{k}\right\|$. Then $\left\|v_{k}\right\|=1$ and $\left\|v_{k}\right\|_{q} \leq \gamma_{q}\left\|v_{k}\right\|=\gamma_{q}$ for $2 \leq q \leq \infty$. Observe that for $k$ large

$$
c+1 \geq \Phi\left(u_{k}\right)-\frac{1}{2}\left\langle\Phi^{\prime}\left(u_{k}\right), u_{k}\right\rangle=\sum_{n \in \mathbb{Z}} \widetilde{W}\left(n, u_{k}(n)\right) .
$$

It follows from (2.4) and (2.8) that

$$
\frac{1}{2} \leq \limsup _{k \rightarrow \infty} \sum_{n \in \mathbb{Z}} \frac{\left|W\left(n, u_{k}(n)\right)\right|}{\left\|u_{k}\right\|^{2}} .
$$

For $0 \leq a<b$, let

$$
\Omega_{k}(a, b)=\left\{n \in \mathbb{Z}: a \leq\left|u_{k}(n)\right|<b\right\} .
$$

Passing to a subsequence, we may assume that $v_{k} \rightarrow v$ in $E$; then $v_{k}(n) \rightarrow v(n)$ for all $n \in \mathbb{Z}$.

Let $\delta:=\lim \sup _{k \rightarrow \infty}\left\|v_{k}\right\|_{\infty}$. By (W1) and (W2), there exist $a_{0} \in\left(0, R_{0}\right)$ and $\Theta>0$ such that

$$
|W(n, x)| \leq \frac{1}{3 \gamma_{2}}|x|^{2}, \quad \forall(n, x) \in \mathbb{Z} \times \mathbb{R}^{\mathcal{N}}, \quad|x| \leq a_{0},
$$

and

$$
|W(n, x)| \leq \Theta|x|^{2}, \quad \forall(n, x) \in \mathbb{Z} \times \mathbb{R}^{\mathcal{N}}, \quad|x| \leq R_{0} ;
$$

consequently,

$$
\sum_{n \in \Omega_{k}\left(0, a_{0}\right)} \frac{\left|W\left(n, u_{k}(n)\right)\right|}{\left|u_{k}(n)\right|^{2}}\left|v_{k}(n)\right|^{2} \leq \frac{1}{3 \gamma_{2}} \sum_{n \in \Omega_{k}\left(0, a_{0}\right)}\left|v_{k}(n)\right|^{2} \leq \frac{1}{3}, \quad k \in \mathbb{N} .
$$

Let

$$
\vartheta=\inf \left\{\frac{\widetilde{W}(n, x)}{|x|^{2}}: n \in \mathbb{Z}, x \in \mathbb{R}^{\mathcal{N}}, a_{0} \leq|x| \leq R_{0}\right\}
$$


Since $W(n, x)$ depends periodically on $n$ and $\widetilde{W}(n, x)>0$ if $x \neq 0$, one has $\vartheta>0$ and

$$
\widetilde{W}\left(n, u_{k}(n)\right) \geq \vartheta\left|u_{k}(n)\right|^{2}, \quad \forall n \in \Omega_{k}\left(a_{0}, R_{0}\right) .
$$

From (W4), (2.9) and (2.14), we have

$$
c+1 \geq \sum_{n \in \mathbb{Z}} \widetilde{W}\left(n, u_{k}(n)\right) \geq \vartheta \sum_{n \in \Omega_{k}\left(a_{0}, R_{0}\right)}\left|u_{k}(n)\right|^{2} .
$$

It follows from (2.12) that

$$
\begin{aligned}
\sum_{n \in \Omega_{k}\left(a_{0}, R_{0}\right)} \frac{\left|W\left(n, u_{k}(n)\right)\right|}{\left|u_{k}(n)\right|^{2}}\left|v_{k}(n)\right|^{2} & \leq \Theta \sum_{n \in \Omega_{k}\left(a_{0}, R_{0}\right)}\left|v_{k}(n)\right|^{2} \\
& =\frac{\Theta}{\left\|u_{k}\right\|^{2}} \sum_{n \in \Omega_{k}\left(a_{0}, R_{0}\right)}\left|u_{k}(n)\right|^{2} \\
& \leq \frac{(c+1) \Theta}{\vartheta\left\|u_{k}\right\|^{2}} \rightarrow 0, \quad k \rightarrow \infty .
\end{aligned}
$$

If $\delta=0$, then from (W4) and (2.9), one has

$$
\begin{aligned}
& \sum_{n \in \Omega_{k}\left(R_{0}, \infty\right)} \frac{\left|W\left(n, u_{k}(n)\right)\right|}{\left|u_{k}(n)\right|^{2}}\left|v_{k}(n)\right|^{2} \\
\leq & \left\|v_{k}\right\|_{\infty} \sum_{n \in \Omega_{k}\left(R_{0}, \infty\right)} \frac{\left|W\left(n, u_{k}(n)\right)\right|}{\left|u_{k}(n)\right|^{2}} \\
\leq & c_{1}\left\|v_{k}\right\|_{\infty} \sum_{n \in \Omega_{k}\left(R_{0}, \infty\right)} \widetilde{W}\left(n, u_{k}(n)\right) \\
\leq & c_{1}(c+1)\left\|v_{k}\right\|_{\infty} \rightarrow 0, \quad k \rightarrow \infty .
\end{aligned}
$$

From (2.13), (2.16) and (2.17), we have

$$
\begin{aligned}
\sum_{n \in \mathbb{Z}} \frac{\left|W\left(n, u_{k}(n)\right)\right|}{\left\|u_{k}\right\|^{2}}= & \sum_{n \in \Omega_{k}\left(0, a_{0}\right)} \frac{\left|W\left(n, u_{k}(n)\right)\right|}{\left|u_{k}(n)\right|^{2}\left|v_{k}(n)\right|^{2}} \\
& +\sum_{n \in \Omega_{k}\left(a_{0}, R_{0}\right)} \frac{\left|W\left(n, u_{k}(n)\right)\right|}{\left|u_{k}(n)\right|^{2}}\left|v_{k}(n)\right|^{2} \\
& +\sum_{n \in \Omega_{k}\left(R_{0}, \infty\right)} \frac{\left|W\left(n, u_{k}(n)\right)\right|}{\left|u_{k}(n)\right|^{2}}\left|v_{k}(n)\right|^{2} \\
& \leq \frac{1}{3}+o(1), \quad k \rightarrow \infty,
\end{aligned}
$$

which contradicts (2.10). Thus $\delta>0$.

Going to a subsequence if necessary, we may assume the existence of $n_{k} \in \mathbb{Z}$ such that

$$
\left|v_{k}\left(n_{k}\right)\right|=\left\|v_{k}\right\|_{\infty}>\frac{\delta}{2} .
$$

Choose integers $i_{k}$ and $m_{k}$ with $0 \leq m_{k} \leq N-1$ such that $n_{k}=i_{k} N+m_{k}$. Let $w_{k}(n)=v_{k}\left(n+i_{k} N\right)$; then

$$
\left|w_{k}\left(m_{k}\right)\right|>\frac{\delta}{2}, \quad \forall k \in N .
$$


Now we define $\tilde{u}_{k}(n)=u_{k}\left(n+i_{k} N\right)$. Since $p(n), L(n)$ and $W(n, x)$ are $N$-periodic on $n$, then $\tilde{u}_{k}(n) /\left\|u_{k}\right\|=w_{k}(n)$ and $\left\|\tilde{u}_{k}\right\|=\left\|u_{k}\right\|$. Passing to a subsequence, we have $w_{k} \rightarrow w$ in $E$; then $w_{k}(n) \rightarrow w(n)$ for all $n \in \mathbb{Z}$. Thus (2.18) implies that $w(n) \neq 0$ for some $n \in\{0,1, \ldots, N-1\}$.

It is obvious that $w(n) \neq 0$ implies $\lim _{k \rightarrow \infty}\left|\tilde{u}_{k}(n)\right|=\infty$. Hence, it follows from (2.4) and (W3) that

$$
\begin{aligned}
0 & =\lim _{k \rightarrow \infty} \frac{c+o(1)}{\left\|u_{k}\right\|^{2}}=\lim _{k \rightarrow \infty} \frac{\Phi\left(u_{k}\right)}{\left\|u_{k}\right\|^{2}} \\
& =\lim _{k \rightarrow \infty}\left[\frac{1}{2}-\sum_{n \in \mathbb{Z}} \frac{W\left(n, u_{k}(n)\right)}{\left|u_{k}(n)\right|^{2}}\left|v_{k}(n)\right|^{2}\right] \\
& =\lim _{k \rightarrow \infty}\left[\frac{1}{2}-\sum_{n \in \mathbb{Z}} \frac{W\left(n+i_{k} N, u_{k}\left(n+i_{k} N\right)\right)}{\left|u_{k}\left(n+i_{k} N\right)\right|^{2}}\left|v_{k}\left(n+i_{k} N\right)\right|^{2}\right] \\
& =\lim _{k \rightarrow \infty}\left[\frac{1}{2}-\sum_{n \in \mathbb{Z}} \frac{W\left(n, \tilde{u}_{k}(n)\right)}{\left|\tilde{u}_{k}(n)\right|^{2}}\left|w_{k}(n)\right|^{2}\right] \\
& \leq \frac{1}{2}-\liminf _{k \rightarrow \infty} \sum_{n \in \mathbb{Z}} \frac{W\left(n, \tilde{u}_{k}(n)\right)}{\left|\tilde{u}_{k}(n)\right|^{2}}\left|w_{k}(n)\right|^{2} \\
& \leq \frac{1}{2}-\liminf _{k \rightarrow \infty} \sum_{n=0}^{N-1} \frac{W\left(n, \tilde{u}_{k}(n)\right)}{\left|\tilde{u}_{k}(n)\right|^{2}}\left|w_{k}(n)\right|^{2} \\
& =-\infty,
\end{aligned}
$$

which is a contradiction. Thus $\left\{u_{k}\right\}$ is bounded in $E$.

\section{Proof of the theOrem}

Proof of Theorem 1.1. Lemma 2.3 implies the existence of a sequence $\left\{u_{k}\right\} \subset E$ satisfying (2.6), and so (2.8). By Lemma 2.4, $\left\{u_{k}\right\}$ is bounded in $E$. Thus there exists a constant $C_{1}>0$ such that

$$
\sqrt[4]{(\beta+4 \alpha) \beta}\left\|u_{k}\right\|_{\infty} \leq\left\|u_{k}\right\| \leq C_{1}, \quad \forall k \in \mathbb{N}
$$

Hence, by (W1) and (W2), there exists a constant $C_{2}>0$ such that

$$
|\widetilde{W}(n, x)| \leq \frac{c}{2 C_{1}^{2} \gamma_{2}^{2}}|x|^{2}+C_{2}|x|^{3}, \quad \forall(n, x) \in \mathbb{Z} \times \mathbb{R}^{\mathcal{N}}, \quad|x| \leq \frac{1}{\sqrt[4]{(\beta+4 \alpha) \beta}} C_{1}
$$

If $\delta:=\lim \sup _{k \rightarrow \infty}\left\|u_{k}\right\|_{\infty}=0$, then for $q>2$,

$$
\sum_{n \in \mathbb{Z}}\left|u_{k}(n)\right|^{q} \leq\left\|u_{k}\right\|_{\infty}^{q-2} \sum_{n \in \mathbb{Z}}\left|u_{k}(n)\right|^{2} \leq \gamma_{2}^{2}\left\|u_{k}\right\|_{\infty}^{q-2} \rightarrow 0, \quad k \rightarrow \infty .
$$


From (2.4), (2.5), (2.8), (3.2) and (3.3), one has

$$
\begin{aligned}
c & =\Phi\left(u_{k}\right)-\frac{1}{2}\left\langle\Phi^{\prime}\left(u_{k}\right), u_{k}\right\rangle+o(1) \\
& =\sum_{n \in \mathbb{Z}} \widetilde{W}\left(n, u_{k}(n)+o(1)\right. \\
& \leq \frac{c}{2 C_{1}^{2} \gamma_{2}^{2}} \sum_{n \in \mathbb{Z}}\left|u_{k}(n)\right|^{2}+C_{2} \sum_{n \in \mathbb{Z}}\left|u_{k}(n)\right|^{3}+o(1) \\
& \leq \frac{c}{2}+o(1), \quad k \rightarrow \infty .
\end{aligned}
$$

This contradiction shows that $\delta>0$.

Going to a subsequence if necessary, we may assume the existence of $n_{k} \in \mathbb{Z}$ such that

$$
\left|u_{k}\left(n_{k}\right)\right|=\left\|u_{k}\right\|_{\infty}>\frac{\delta}{2} .
$$

Choose integers $i_{k}$ and $m_{k}$ with $0 \leq m_{k} \leq N-1$ such that $n_{k}=i_{k} N+m_{k}$. Let $v_{k}(n)=u_{k}\left(n+i_{k} N\right)$; then

$$
\left|v_{k}\left(m_{k}\right)\right|>\frac{\delta}{2}, \quad \forall k \in \mathbb{N} .
$$

Since $p(n), L(n)$ and $W(n, x)$ are $N$-periodic on $n$, we have $\left\|v_{k}\right\|=\left\|u_{k}\right\|$ and

$$
\Phi\left(v_{k}\right) \rightarrow c, \quad\left\|\Phi^{\prime}\left(v_{k}\right)\right\|\left(1+\left\|v_{k}\right\|\right) \rightarrow 0 .
$$

Passing to a subsequence, we have $v_{k} \rightarrow v$ in $E, v_{k}(n) \rightarrow v(n)$ for all $n \in \mathbb{Z}$. Thus, (3.4) implies that $v \neq 0$. Let

$$
E_{0}=\{u \in E:\{n \in \mathbb{Z}:|u(n)|>0\} \text { is finite set }\} .
$$

Then for every $w \in E_{0}$, there exists an $n_{0} \in \mathbb{N}$ such that $w(n)=0$ for all $|n|>n_{0}$. Hence, it follows from (2.5) and (3.5) that

$$
\begin{aligned}
\left\langle\Phi^{\prime}(v), w\right\rangle= & \sum_{n \in \mathbb{Z}}[(p(n+1) \triangle v(n), \triangle w(n))+(L(n) v(n), w(n)) \\
& -(\nabla W(n, v(n)), w(n))] \\
= & \sum_{|n| \leq n_{0}}[(p(n+1) \triangle v(n), \triangle w(n))+(L(n) v(n), w(n)) \\
=\lim _{k \rightarrow \infty} \sum_{|n| \leq n_{0}}\left[\left(p(n+1) \triangle v_{k}(n), \triangle w(n)\right)+\left(L(n) v_{k}(n), w(n)\right)\right. & \left.-\left(\nabla W\left(n, v_{k}(n)\right), w(n)\right)\right] \\
=\lim _{k \rightarrow \infty}\left\langle\Phi^{\prime}\left(v_{k}\right), w\right\rangle=0 . &
\end{aligned}
$$

Since $E_{0}$ is dense in $E, \Phi^{\prime}(v)=0$. Thus the proof is finished.

\section{REFERENCES}

[1] Ravi P. Agarwal, Difference equations and inequalities: Theory, methods, and applications, 2nd ed., Monographs and Textbooks in Pure and Applied Mathematics, vol. 228, Marcel Dekker Inc., New York, 2000. MR.1740241 (2001f:39001)

[2] Calvin D. Ahlbrandt and Allan C. Peterson, Discrete Hamiltonian systems: Difference equations, continued fractions, and Riccati equations, Kluwer Texts in the Mathematical Sciences, vol. 16, Kluwer Academic Publishers Group, Dordrecht, 1996. MR.1423802 (98m:39043) 
[3] Giovanna Cerami, An existence criterion for the critical points on unbounded manifolds (Italian), Istit. Lombardo Accad. Sci. Lett. Rend. A 112 (1978), no. 2, 332-336 (1979). MR.581298 (81k:58021)

[4] Xiaoqing Deng and Gong Cheng, Homoclinic orbits for second order discrete Hamiltonian systems with potential changing sign, Acta Appl. Math. 103 (2008), no. 3, 301-314, DOI 10.1007/s10440-008-9237-z. MR2430446 (2009f:39012)

[5] Xiaoqing Deng, Gong Cheng, and Haiping Shi, Subharmonic solutions and homoclinic orbits of second order discrete Hamiltonian systems with potential changing sign, Comput. Math. Appl. 58 (2009), no. 6, 1198-1206, DOI 10.1016/j.camwa.2009.06.045. MR2554352 (2010i:37145)

[6] Yan Heng Ding, Existence and multiplicity results for homoclinic solutions to a class of Hamiltonian systems, Nonlinear Anal. 25 (1995), no. 11, 1095-1113, DOI 10.1016/0362546X(94)00229-B. MR1350732 (96g:34070)

[7] Manjun Ma and Zhiming Guo, Homoclinic orbits for second order self-adjoint difference equations, J. Math. Anal. Appl. 323 (2006), no. 1, 513-521, DOI 10.1016/j.jmaa.2005.10.049. MR2262222 (2007f:37093)

[8] Manjun Ma and Zhiming Guo, Homoclinic orbits and subharmonics for nonlinear second order difference equations, Nonlinear Anal. 67 (2007), no. 6, 1737-1745, DOI 10.1016/j.na.2006.08.014. MR2326026 (2008f:37133)

[9] Xiaoyan Lin and X. H. Tang, Existence of infinitely many homoclinic orbits in discrete Hamiltonian systems, J. Math. Anal. Appl. 373 (2011), no. 1, 59-72, DOI 10.1016/j.jmaa.2010.06.008. MR2684457(2011i:39019)

[10] W. Omana and M. Willem, Homoclinic orbits for a class of Hamiltonian systems, Differential Integral Equations 5 (1992), no. 5, 1115-1120. MR.1171983 (93f:58031)

[11] Z. Q. Ou and C. L. Tang, Existence of homoclinic orbits for the second order Hamiltonian systems, J. Math. Anal. Appl. 291(1) (2004), 203-213.

[12] X. H. Tang, Xiaoyan Lin, and Li Xiao, Homoclinic solutions for a class of second order discrete Hamiltonian systems, J. Difference Equ. Appl. 16 (2010), no. 11, 1257-1273, DOI 10.1080/10236190902791635. MR2738948 (2012f:39009)

[13] X. H. Tang and Xiaoyan Lin, Existence and multiplicity of homoclinic solutions for secondorder discrete Hamiltonian systems with subquadratic potential, J. Difference Equ. Appl. 17 (2011), no. 11, 1617-1634, DOI 10.1080/10236191003730514. MR2846503 (2012h:70032)

[14] Xian Hua Tang and Xiao Yan Lin, Homoclinic solutions for a class of second order discrete Hamiltonian systems, Acta Math. Sin. (Engl. Ser.) 28 (2012), no. 3, 609-622, DOI 10.1007/s10114-012-9233-0. MR2891289

[15] X. H. Tang and X. Y. Lin, Infinitely many homoclinic orbits for discrete Hamiltonian systems with subquadratic potential, J. Difference. Equ. Appl. 19 (2013), no. 5, 796-813. DOI:10.1080/10236198.2012.691168. MR349055

[16] X. H. Tang and Li Xiao, Homoclinic solutions for ordinary p-Laplacian systems with a coercive potential, Nonlinear Anal. 71 (2009), no. 3-4, 1124-1132, DOI 10.1016/j.na.2008.11.027. MR2527532(2010h:37144)

[17] Jianshe Yu, Haiping Shi, and Zhiming Guo, Homoclinic orbits for nonlinear difference equations containing both advance and retardation, J. Math. Anal. Appl. 352 (2009), no. 2, 799-806, DOI 10.1016/j.jmaa.2008.11.043. MR2501925 (2009m:39025)

[18] Zhan Zhou, JianShe Yu, and YuMing Chen, Homoclinic solutions in periodic difference equations with saturable nonlinearity, Sci. China Math. 54 (2011), no. 1, 83-93, DOI 10.1007/s11425-010-4101-9. MR2764787(2011m:39013)

College of Mathematics and Information Sciences, Guangzhou University, GuangZHOU 510006, PeOple's Republic of China

E-mail address: qinqin.zhang0413@gmail.com 